\section{PREVALÊNCIA DE COMPULSÃO ALIMENTAR PERIÓDICA E AVALIAÇÃO DO CONSUMO ALIMENTAR DE INDIVÍDUOS COM EXCESSO DE PESO}

\author{
Prevalence of binge eating disorder and assessment of food \\ consumption in people with excess weight
}

\section{Prevalencia de compulsión alimentaria periódica y valoración del consumo alimentario de individuos con exceso de peso}

\section{RESUMO}

Objetivo: Verificar a prevalência de compulsão alimentar periódica e avaliar o consumo alimentar de indivíduos com excesso de peso. Métodos: Trata-se de um estudo observacional, de caráter transversal, realizado com pacientes com IMC igual ou superior a $25 \mathrm{Kg} / \mathrm{m}^{2}$, pertencentes à faixa etária de 20 a 59 anos, atendidos nas duas Unidades de Saúde da Família do centro da cidade de Quixeré-CE. A amostra consistiu de 100 pessoas. Foi realizada uma entrevista para triagem da compulsão alimentar periódica. Os participantes responderam a um questionário sobre aspectos socioeconômicos, foram submetidos a uma avaliação antropométrica (peso, altura) e a ingestão alimentar foi avaliada com base em dois recordatórios alimentares. Resultados: Entre os entrevistados, 3\% foram diagnosticados com CAP grave, $20 \%$ com CAP moderada e $77 \%$ não tinham Compulsão Alimentar Periódica. Pacientes com graus mais elevados de CAP apresentaram-se com maior excesso ponderal $(\mathrm{p}=0,03)$. Os entrevistados com a compulsão moderada e grave, em comparação àqueles sem CAP, mostraram ter um consumo alimentar maior e em desequilíbrio, em se tratando de qualidade de consumo alimentar, para calorias e fibras ( $\mathrm{p}=0,000$ e $\mathrm{p}=0,047$, respectivamente). Conclusão: Um terço dos participantes apresentou algum grau de compulsão alimentar, relacionado com alteração de estado nutricional, destacando que os participantes com compulsão alimentar periódica moderada e grave apresentaram um padrão alimentar com mais calorias e menos fibra.

Descritores: Comportamento Compulsivo; Obesidade; Consumo de Alimentos.

Objective: To determine the prevalence of binge eating disorder and assess food consumption in individuals with excess weight. Methods: This is an observational crosssectional study conducted with patients with BMI equal to or greater than $25 \mathrm{~kg} / \mathrm{m}^{2}$, aged 20 to 59 years, attending two Family Health Care Centers in the heart of the city of Quixeré, Ceará. The sample comprised 100 people. An interview was carried out in order to screen for binge eating disorder. Participants answered a questionnaire on socioeconomic aspects, underwent anthropometric (weight, height) assessment, and food consumption was assessed based on two dietary recalls. Results: Among respondents, 3\% were diagnosed with severe BED, $20 \%$ with moderate BED and $77 \%$ did not have Binge Eating Disorder. Patients with higher degrees of BED presented greater excess weight, which as not statistically significant $(p=0.257)$. Respondents with moderate and severe binge eating disorder, when compared to those without BED, presented a greater and unbalanced food consumption in terms of quality of food consumption; however, this was not significant (p ranging from 0.171 to 0.567 ). Conclusion: One third of participants had some degree of binge eating disorder, which was not related to changes in nutritional status, although participants with moderate and severe binge eating disorder showed a dietary pattern with more calories and less fiber.

Descriptors: Compulsive Behavior; Obesity; Food Consumption.

Artigo Original
1) Instituto Federal de Educação, Ciência e Tecnologia do Ceará - IFCE - Limoeiro do Norte (CE) - Brasil
Recebido em: 11/07/2016

Revisado em: 03/08/2016

Aceito em: 20/09/2016 


\section{RESUMEN}

Objetivo: Verificar la prevalencia de compulsión alimentaria periódica (CAP) y valorar el consumo alimentario de individuos con exceso de peso. Métodos: Se trata de un estudio observacional de carácter transversal realizado con pacientes con el IMC igual o mayor que $25 \mathrm{Kg} / \mathrm{m}^{2}$ en la franja de edad entre 20 y 59 años asistidos en las dos Unidades de Salud de la Familia del centro de la ciudad de Quixeré-Ceará. La muestra consistió de 100 personas. Se realizó una entrevista la para selección de la compulsión alimentaria periódica. Los participantes contestaron a una encuesta sobre los aspectos socioeconómicos y participaron de una evaluación antropométrica (peso, altura) y la ingesta de alimentos fue valorada basándose en dos recordatorios de alimentos. Resultados: El 3\% de los entrevistados recibió el diagnóstico de CAP grave, el 20\% de CAP moderada y el 77\% no presentaron Compulsión Alimentaria Periódica. Los pacientes con grados más elevados de CAP presentaron más exceso ponderal estadisticamente no significativo $(p=0,257)$. Los entrevistados con compulsión de moderada a grave comparados a los sin CAP, presentaron mayor consumo de alimentos y en desequilibrio respecto la calidad del consumo de alimentos pero no significativo (variación de $p$ entre 0,171 y 0,567). Conclusión: Un tercio de los participantes presentó algún grado de compulsión alimentaria sin relación con la alteración del estado nutricional aunque los participantes con compulsión alimentaria periódica moderada y grave presentaron un patrón alimentario con más calorías y menos fibras.

Descriptores: Conducta Compulsiva; Obesidad; Consumo de Alimentos.

\section{INTRODUÇÃO}

A obesidade é uma doença crônica, multifatorial e que representa fator de risco para diversas outras morbidades. É determinada pela associação de vários fatores, dentre os quais se pode enumerar os orgânicos, genéticos, ambientais, culturais e de estilo de vida, como os alimentares e o sedentarismo ${ }^{(1)}$.

Nos últimos anos, essa doença tornou-se foco de estudo entre os pesquisadores devido ao aumento alarmante de indivíduos com sobrepeso e obesidade, principalmente em países desenvolvidos ${ }^{(1)}$.

Além de estar frequentemente associada a doenças físicas e metabólicas, a obesidade está diretamente relacionada com o consumo alimentar inadequado, que associado com a falta de exercício físico, resulta em um balanço energético positivo, em que o consumo de energia é maior que o gasto, ocasionando assim a obesidade ${ }^{(2,3)}$. Vale ressaltar que além da manutenção do peso corporal, a prática regular de atividade física contribui ainda com a prevenção de doenças cardiovasculares e osteoarticulares e controle da pressão arterial e dos níveis de colesterol ${ }^{(4)}$.

O consumo alimentar inadequado consiste, principalmente, na ingestão exagerada de açúcar simples, gorduras saturadas e proteínas, com baixo consumo de fibras. Insere-se ainda neste consumo inadequado e, portanto, entre as causas da obesidade, aquele decorrente da presença de Compulsão Alimentar Periódica (CAP) ou do Transtorno de Compulsão Alimentar Periódica (TCAP). A primeira consiste na ingestão, em um período de até duas horas, de uma quantidade de alimentos maior do que a maioria das pessoas consumiria durante um período similar e sob circunstâncias semelhantes, estando associada a um sentimento de falta de controle sobre o comportamento alimentar. Para caracterizá-la como transtorno (TCAP), é necessário, além dos critérios de CAP, que o episódio ocorra pelo menos dois dias na semana nos últimos seis meses, associado a características de perda de controle e sem comportamento compensatório para a perda de peso ${ }^{(5,6)}$.

A prevalência da compulsão alimentar periódica pode variar de 1,5 a $5 \%$ na população geral, portadora ou não de excesso de peso. Já entre indivíduos em tratamento clínico para perda de peso, a prevalência varia de 5 a $30 \%{ }^{(7)}$. Quanto à TCAP, um primeiro trabalho mostra que a prevalência é de $2 \%$ para a população geral e $30 \%$ para obesos em tratamento ${ }^{(7)}$. Já um segundo trabalho sugere que essa prevalência é de $3 \%$ para a população e varia de 7,5 a $46 \%$ entre os obesos ${ }^{(8)}$. No Brasil, dos pacientes que procuram tratamento para emagrecer, estima-se que $15 \%$ a 22\% apresentam TCAP. Já entre aqueles pacientes que realizam cirurgia bariátrica o transtorno pode acometer $56 \%$ dos indivíduos ${ }^{(9)}$.

Assim, sabendo-se das evidências de prevalência significativa de CAP e TCAP na população com sobrepeso ou obesidade e que em decorrência do excesso de peso, pode haver complicações à saúde, visou-se realizar este estudo nas Unidades de Saúde da Família da cidade de Quixeré-CE. A escolha desses locais deveu-se ao fato de que, empiricamente, observa-se que estes serviços recebem um número elevado de indivíduos com excesso de peso ou comorbidades dele resultantes, como hipertensão e diabetes, existindo inclusive dias de atendimento exclusivos para esse público.

Vale ressaltar a relevância deste estudo, pois, uma vez identificada à compulsão na população avaliada, os gestores de saúde do município poderão intervir por meio do incentivo à adoção de um estilo de vida saudável, visando deter o excesso ponderal e as complicações oriundas da obesidade, podendo melhorar a qualidade de vida destes.

Diante do exposto, o presente trabalho teve por objetivo verificar a prevalência de compulsão alimentar periódica e 
avaliar o consumo alimentar de indivíduos com excesso de peso.

\section{MÉTODOS}

Trata-se de um estudo observacional, de caráter transversal, realizado com pacientes que apresentavam Índice de Massa Corporal (IMC) superior ou igual a $25 \mathrm{Kg} /$ $\mathrm{m}^{2}$, com faixa etária de 20 a 59 anos $^{(10)}$, não possuindo diagnóstico de doença que influencie no ganho de peso e que eram atendidos nas duas Unidades de Saúde da Família do centro da cidade de Quixeré-CE. A pesquisa ocorreu no período de outubro de 2014 a março de 2015, com turno de coleta variando entre manhã e tarde.

Para seleção da amostra, foram utilizados dados fornecidos pela coordenação das Unidades de Saúde da Família. O número amostral foi calculado com margem de erro de $5 \%$, tomando como base um tamanho populacional de 3086 indivíduos, obtendo-se assim, um valor correspondente a 100 pacientes $^{(11)}$.

Para a realização da triagem da CAP entre os pacientes, foi aplicada, por meio de entrevista, a Escala de Compulsão Alimentar Periódica (ECAP) ${ }^{(12)}$. Utilizou-se ainda um questionário elaborado pelo próprio pesquisador visando obter dados pessoais do entrevistado, informações antropométricas e socioeconômicas, bem como a investigação acerca de doenças que podiam resultar em ganho de peso e da prática de atividade física. Foram ainda coletados dois recordatórios alimentares de 24 horas, que são considerados padrão-ouro para estimar o consumo alimentar habitual se aplicados de maneira seriada, em dias não consecutivos, incluindo um dia fim de semana. A partir desses instrumentos puderam ser quantificados, a partir de medidas caseiras, alimentos e bebidas consumidos no dia anterior à aplicação ${ }^{(13)}$.

Os dados antropométricos foram coletados conforme recomendado pela literatura ${ }^{(14)}$. Assim, para aferição do peso corporal, foi solicitado que os entrevistados permanecessem descalços e que se posicionassem sobre uma balança eletrônica do tipo plataforma, da marca Balmak ${ }^{\circledR}$, com capacidade para $200 \mathrm{~kg}$ e graduação de 100 gramas. Já para a medida da altura, foi utilizado um estadiômetro compacto da marca MD HealthCare ${ }^{\circledR}$, com escala em milímetros. Os pacientes mantiveram-se descalços, com os pés juntos, calcanhares encostados na parede, em postura ereta, com olhar fixo no horizonte, para que fosse efetuada a leitura. Os dados de peso e altura subsidiaram o cálculo do IMC, a partir do qual se categorizou o estado nutricional individual, com base nos pontos de corte estabelecidos pela Organização Mundial de Saúde ${ }^{(14,15)}$.

Os dados foram coletados em medidas caseiras, a partir da aplicação dos recordatórios, e convertidos em gramas ${ }^{(16)}$, sendo então utilizados para análise da composição nutricional ${ }^{(17)}$. Foi considerado consumo habitual de calorias e nutrientes a média dos valores obtidos para cada indivíduo a partir dos dois recordatórios registrados.

Para análise dos dados, calculou-se média e desvio padrão para as variáveis contínuas, enquanto as categóricas foram descritas por frequência absoluta e relativa, em números e percentuais. Com o auxílio do software SPSS versão 20, utilizou-se o teste de t de Student para comparar as médias de IMC e de consumo de nutrientes dos entrevistados conforme o diagnóstico de CAP. Adotou-se p $<0,05$ como nível de significância.

Este estudo foi submetido ao Comitê de Ética em Pesquisa do Instituto Federal de Educação, Ciências e Tecnologia do Ceará, campus Fortaleza, e que o mesmo seguiu o preconizado pela resolução n. ${ }^{\circ} 466 / 12$ do Conselho Nacional de Saúde (CNS), referente a pesquisas envolvendo seres humanos, sob o parecer de $\mathrm{n}^{\mathrm{o}}$. 787.817.

\section{RESULTADOS}

A amostra foi composta por 100 pessoas, em sua maioria do sexo feminino $(76,00 \%, \mathrm{n}=76)$. A faixa etária variou de 21 a 59 anos, prevalecendo à faixa de 30 a 49 anos, com $62,00 \%$ ( $\mathrm{n}=62$ ) (média de 41,12 $\pm 10,40$ anos). Percebeuse ainda que haja maior percentual de mulheres praticantes de atividade física $(36,84 \%, \mathrm{n}=28)$ quando comparadas aos homens $(25 \%, \mathrm{n}=6)$, conforme demonstrado na tabela I.

Quanto à renda mensal, a média foi de $\mathrm{R} \$ 906,32 \pm$ $\mathrm{R} \$ 1021,99$, e a maior parte das pessoas $(64,00 \%, \mathrm{n}=64)$ relatou receber menos de 1 salário mínimo. Com relação ao estado nutricional, a maioria dos entrevistados $(58,00 \%$, $\mathrm{n}=58$ ) apresentou o diagnóstico de sobrepeso, com média do IMC de 30,00 $\pm 3,80 \mathrm{Kg} / \mathrm{m}^{2}$. Esse dado se confirmou quando se separou por sexo, em que $60,53 \%(n=46)$ das mulheres e $50,00 \%(n=12)$ dos homens apresentaram esse estado nutricional.

Do total de participantes, $3,00 \% \quad(n=3)$ foram diagnosticados com CAP grave, 20,00\% ( $\mathrm{n}=20)$ com CAP moderada e $77,00 \%(n=77)$ não tinham Compulsão Alimentar Periódica. Daqueles indivíduos que apresentaram CAP grave, $66,67 \%(n=2)$ foram diagnosticados como sobrepesados e $33,33 \%(n=1)$ como obesos de nível I. Em todas as categorias de estado nutricional investigadas houve prevalência de indivíduos sem CAP, à exceção dos obesos grau II, visto que $66,67 \%(n=4)$ deles tinham CAP moderada (Tabela II).

De acordo com a tabela II, percebeu-se que as médias de IMC foram maiores nos entrevistados com CAP moderada e grave, com 31,91 $\pm 4,17$ e 29,92 $\pm 1,69$ respectivamente, quando comparado aos sem $\mathrm{CAP}(\mathrm{p}=0,03)$. 
Tabela I - Descrição sociodemográfica, do estado nutricional e de estilo de vida de indivíduos com excesso de peso. Quixeré, Ceará, 2015.

\begin{tabular}{|c|c|c|c|c|c|c|c|c|}
\hline \multirow{3}{*}{ Variáveis } & \multicolumn{4}{|c|}{ Sexo } & \multirow{2}{*}{\multicolumn{2}{|c|}{ Total }} & \multirow{3}{*}{ Média } & \multirow{3}{*}{ p-valor } \\
\hline & \multicolumn{2}{|c|}{ Masculino } & \multicolumn{2}{|c|}{ Feminino } & & & & \\
\hline & $\mathrm{n}$ & $\%$ & $\mathbf{n}$ & $\%$ & $\mathbf{n}$ & $\%$ & & \\
\hline \multicolumn{9}{|l|}{ Idade (Anos) } \\
\hline$<30$ & 4 & 16,67 & 10 & 13,16 & 14 & 14,00 & \multirow{3}{*}{41,12} & \multirow{3}{*}{10,40} \\
\hline$\geq 30 \mathrm{a}<50$ & 15 & 62,50 & 47 & 61,84 & 62 & 62,00 & & \\
\hline$\geq 50$ & 5 & 20,83 & 19 & 25,00 & 24 & 24,00 & & \\
\hline \multicolumn{9}{|l|}{ Renda } \\
\hline <1 salário mínimo & 7 & 29,17 & 57 & 75,00 & 64 & 64,00 & \multirow{3}{*}{906,32} & \multirow{3}{*}{1021,99} \\
\hline 1 a 2 salários mínimo & 10 & 41,67 & 14 & 18,42 & 24 & 24,00 & & \\
\hline >2 salários mínimo & 7 & 29,17 & 5 & 6,58 & 12 & 12,00 & & \\
\hline \multicolumn{9}{|l|}{ Atividade Física } \\
\hline Sim & 6 & 25,00 & 28 & 36,84 & 34 & 34,00 & \multirow[b]{2}{*}{-} & \\
\hline Não & 18 & 75,00 & 48 & 63,16 & 66 & 66,00 & & \\
\hline \multicolumn{9}{|l|}{ Estado Nutricional\# } \\
\hline Sobrepeso & 12 & 50,00 & 46 & 60,53 & 58 & 58,00 & \multirow{4}{*}{$30,00^{*}$} & \multirow{4}{*}{$3,80^{*}$} \\
\hline Obesidade I & 9 & 37,50 & 22 & 28,95 & 31 & 31,00 & & \\
\hline Obesidade II & 2 & 8,33 & 4 & 5,26 & 6 & 6,00 & & \\
\hline Obesidade III & 1 & 4,17 & 4 & 5,26 & 5 & 5,00 & & \\
\hline Total & 24 & 100,00 & 76 & 100,00 & 100 & 100,00 & & \\
\hline
\end{tabular}

*Calculados com base no índice de massa corporal (IMC). DP: desvio-padrão.

Tabela II - Distribuição dos indivíduos com excesso ponderal conforme o diagnóstico de compulsão alimentar periódica (CAP). Quixeré, Ceará, 2015.

\begin{tabular}{|c|c|c|c|c|c|c|c|c|c|c|c|c|}
\hline \multirow{3}{*}{ CAP } & \multicolumn{8}{|c|}{ Excesso de peso } & \multirow{2}{*}{\multicolumn{2}{|c|}{ Total }} & \multirow{3}{*}{ Média $^{\#}$} & \multirow{3}{*}{ DP } \\
\hline & \multicolumn{2}{|c|}{ Sobrepeso } & \multicolumn{2}{|c|}{ Obesidade I } & \multicolumn{2}{|c|}{ Obesidade II } & \multicolumn{2}{|c|}{ Obesidade III } & & & & \\
\hline & $\mathbf{n}$ & $\%$ & $\mathrm{n}$ & $\%$ & $\mathrm{n}$ & $\%$ & $\mathbf{n}$ & $\%$ & $n$ & $\%$ & & \\
\hline Sem CAP & 47 & 81,03 & 24 & 77,42 & 2 & 33,33 & 4 & 80,00 & 77 & 77,00 & 29,51 & 3,59 \\
\hline Moderada & 9 & 15,52 & 6 & 19,35 & 4 & 66,67 & 1 & 20,00 & 20 & 20,00 & 31,91 & 4,17 \\
\hline Grave & 2 & 3,45 & 1 & 3,23 & 0 & 0,00 & 0 & 0,00 & 3 & 3,00 & 29,92 & 1,69 \\
\hline Total & 58 & 100 & 31 & 100 & 6 & 100 & 5 & 100 & 100 & 100 & - & - \\
\hline
\end{tabular}

\#Calculados com base no IMC. CAP: Compulsão Alimentar Periódica, DP: Desvio-padrão.

${ }^{*} \mathrm{p}<0,05$ ao comparar as médias de peso em cada grupo CAP $(\mathrm{p}=0,030)$.

Em relação ao consumo alimentar, $66,67 \%(\mathrm{n}=2)$ das pessoas que apresentaram CAP grave e $70 \%(n=14)$ dos que apresentaram CAP moderada relataram consumir mais de $2.000 \mathrm{Kcal} / \mathrm{dia}$. Por outro lado, aqueles entrevistados sem CAP estão inseridos na faixa de menor consumo calórico, uma vez que $48,05 \%(n=37)$ apresentaram consumo inferior a $1.500 \mathrm{Kcal} / \mathrm{dia}$.

De acordo com a análise dos recordatórios (Tabela III), foi possível perceber que a maioria dos indivíduos sem CAP $(87,01 \%, n=67)$, com CAP moderada $(85 \%, n=17)$ e todos aqueles com CAP grave apresentaram um consumo de lipídeos dentro do preconizado. Entretanto, as médias de consumo de lipídeos em relação ao VCT apresentaramse maiores para os sujeitos com CAP moderada $(28,28 \pm$ $7,79)$ e grave $(27,94 \pm 4,81)$ quando comparada à média da população em geral $(26,88 \pm 7,07)$.

Em relação ao consumo de carboidratos, grande parte dos entrevistados apresentou um consumo dentro dos limites recomendado, conforme está descrito na tabela III. Todavia, quando se trata do consumo diário de fibras, observou-se que grande parte das pessoas demostraram ingerir menos de $20 \mathrm{~g} /$ dia, sejam elas diagnosticadas com CAP moderada ou grave, com um percentual de $70,00 \%(\mathrm{n}=14)$ e $66,67 \%$ $(n=2)$ respectivamente, ou sem a presença da compulsão alimentar periódica de $83,12 \%(\mathrm{n}=64)$. 
Tabela III - Consumo alimentar dos indivíduos conforme diagnóstico de compulsão alimentar periódica (CAP). Quixeré, Ceará, 2015.

\begin{tabular}{|c|c|c|c|c|c|c|c|c|c|c|c|}
\hline \multirow{3}{*}{$\begin{array}{l}\text { Consumo } \\
\text { alimentar }\end{array}$} & \multicolumn{6}{|c|}{ CAP } & \multirow{2}{*}{\multicolumn{2}{|c|}{ Total }} & \multirow{3}{*}{ Média } & \multirow{3}{*}{ DP } & \multirow{3}{*}{ p-valor } \\
\hline & \multicolumn{2}{|c|}{ Sem CAP } & \multicolumn{2}{|c|}{ Moderada } & \multicolumn{2}{|c|}{ Grave } & & & & & \\
\hline & n & $\%$ & $n$ & $\%$ & $\mathbf{n}$ & $\%$ & $n$ & $\%$ & & & \\
\hline Calorias (Kcal) & & & & & & & & & & & \\
\hline$<1500$ & 37 & 48,05 & 2 & 10,00 & 1 & 33,33 & 40 & 40,00 & & & 0,001 \\
\hline 1500 a 2000 & 25 & 32,47 & 4 & 20,00 & 0 & 0,00 & 29 & 29,00 & $1.771,0$ & 644,48 & \\
\hline$>2000$ & 15 & 19,48 & 14 & 70,00 & 2 & 66,67 & 31 & 31,00 & & & \\
\hline Média & \multicolumn{2}{|c|}{1609,90} & \multicolumn{2}{|c|}{2345,55} & \multicolumn{2}{|c|}{2076,13} & & & & & \\
\hline DP & \multicolumn{2}{|c|}{535,91} & \multicolumn{2}{|c|}{687,50} & \multicolumn{2}{|c|}{602,30} & & & & & \\
\hline \multicolumn{12}{|l|}{ Lipídeos\# } \\
\hline$<10 \%$ & 2 & 2,60 & 0 & 0,00 & 0 & 0,00 & 2 & 2,00 & & & 0,305 \\
\hline $10-35 \%$ & 67 & 87,01 & 17 & 85,00 & 3 & 100,00 & 87 & 87,00 & 26,8 & 7,07 & \\
\hline$>35 \%$ & 8 & 10,39 & 3 & 15,00 & 0 & 0,00 & 11 & 11,00 & & & \\
\hline Média & \multicolumn{2}{|c|}{26,47} & \multicolumn{2}{|c|}{28,26} & \multicolumn{2}{|c|}{27,94} & & & & & \\
\hline DP & \multicolumn{2}{|c|}{6,89} & \multicolumn{2}{|c|}{7,79} & \multicolumn{2}{|c|}{4,81} & & & & & \\
\hline \multicolumn{12}{|l|}{ Carboidratos\# } \\
\hline$<45 \%$ & 5 & 6,49 & 3,00 & 15,00 & 0 & 0,00 & 8 & 8,00 & & & 0,503 \\
\hline $45-65 \%$ & 65 & 84,42 & 14,00 & 70,00 & 3 & 100,00 & 82 & 82,00 & 54,97 & 7,79 & \\
\hline$>65 \%$ & 7 & 9,09 & 3,00 & 15,00 & 0 & 0,00 & 10 & 10,00 & & & \\
\hline Média & \multicolumn{2}{|c|}{55,27} & \multicolumn{2}{|c|}{53,58} & \multicolumn{2}{|c|}{56,44} & & & & & \\
\hline DP & \multicolumn{2}{|c|}{7,65} & \multicolumn{2}{|c|}{8,53} & \multicolumn{2}{|c|}{4,26} & & & & & \\
\hline Fibras & & & & & & & & & & & \\
\hline$<20 \mathrm{~g}$ & 64 & 83,12 & 14,00 & 70,00 & 2 & 66,67 & 80 & 80,00 & & & 0,047 \\
\hline $20-30 \mathrm{~g}$ & 13 & 16,88 & 4,00 & 20,00 & 1 & 33,33 & 18 & 18,00 & 15,10 & 5,86 & \\
\hline$>30 \mathrm{~g}$ & 0 & 0,00 & 2,00 & 10,00 & 0 & 0,00 & 2 & 2,00 & & & \\
\hline Média & & 37 & & & & 28 & & & & & \\
\hline DP & & 43 & & & & 51 & & & & & \\
\hline Total & 77 & 100 & 20 & 100 & 3 & 100 & 100 & 100 & - & - & \\
\hline
\end{tabular}

CAP: Compulsão Alimentar Periódica, DP: Desvio-padrão. \#obtido a partir do Valor Calórico Total (VCT).

Percebeu-se que as médias de consumo de calorias $(\mathrm{p}=0,000)$ e fibras $(\mathrm{p}=0,047)$ diferiram entre pessoas com e sem CAP, mas não para lipídios e carboidratos, em relação ao $\mathrm{VCT}$ (respectivamente, $\mathrm{p}=0,305 \mathrm{e} \mathrm{p}=0,503$ ).

\section{DISCUSSÃO}

Antes de iniciar as discussões acerca dos resultados encontrados, considera-se necessário relembrar que CAP consiste na ingestão de uma quantidade de alimento maior do que a maioria das pessoas conseguiria consumir, em um período de até duas horas, associado a um sentimento de falta de controle em relação ao comportamento alimentar. Caso o episódio ocorra em pelo menos dois dias na semana nos últimos seis meses, sem comportamento compensatório para a perda de peso e associado a características de perda de controle, passa a ser caracterizado como $\operatorname{TCAP}^{(5,6)}$.Vale salientar que para comparação com os achados do atual estudo, foram utilizados trabalhos sobre TCAP, pois ainda são escassas literaturas nacionais e internacionais que abordem a compulsão alimentar como tema principal.

Voltando-se então para a presente pesquisa, ela foi composta principalmente por pessoas do sexo feminino com uma faixa etária média de 41,12 anos. Esses dados são semelhantes ao encontrado num estudo que analisou a possível relação entre a ocorrência de Transtorno do Apetite e Transtorno de Compulsão Alimentar Periódica, realizado com pacientes encaminhados ao ambulatório de Endocrinologia do Hospital das Clínicas de Porto Alegre ${ }^{(18)}$, cujos resultados evidenciaram que $78,80 \%$ dos pacientes eram do sexo feminino e apresentavam idade média de 44,50 anos.

Em um trabalho realizado com indivíduos de 20 a 59 anos de idade da zona urbana de Feira de Santana-BA ${ }^{(19)}$, com o objetivo de estimar a prevalência de comportamentos sugestivos de transtornos alimentares (Bulimia nervosa e TCAP), detectou-se que $73,5 \%$ dos entrevistados eram do sexo feminino, fato este que poderia ser explicado pela 
preocupação com a imagem corporal comumente encontrada nesse público, tornando-as vulneráveis ao desenvolvimento do transtorno.

Em se tratando da renda mensal pessoal, houve predomínio de pessoas na categoria com menos de um salário mínimo por mês. Sobre isso, tem-se verificado que indivíduos que apresentam nível socioeconômico mais baixo podem ter condições de bem-estar físico-mental afetadas $^{(20)}$, implicando em casos de insegurança e estresse, que por sua vez, podem vir a intensificar a frequência dos episódios da compulsão alimentar.

Um estudo de revisão analisou pesquisas latinoamericanas que incluíram indivíduos que apresentavam transtornos e que preenchiam os critérios de TCAP segundo o Diagnostic and Statistical Manual of Mental Disorders (DSM-IV $)^{(21)}$, detectou-se que o IMC médio dos participantes dos estudos analisados correspondeu a $30,1 \mathrm{Kg} / \mathrm{m}^{2}$, estando então próximo ao encontrado no atual trabalho. Acerca disso, outras literaturas observaram que indivíduos com diagnóstico de obesidade se mostraram mais susceptíveis ao desenvolvimento dos episódios compulsivos quando comparados àqueles eutróficos ${ }^{(19)}$.

De fato, é de conhecimento que a prevalência da TCAP é maior naqueles indivíduos que apresentam um IMC mais elevado, uma vez que sua presença tem sido associada com o aumento da adiposidade ${ }^{(22)}$.

A literatura tem descrito que pessoas diagnosticadas com CAP moderada e grave apresentam graus mais severos de excesso ponderal, conforme retratado pelo IMC. Neste estudo, especificamente, em todas as classes de estado nutricional, prevaleceu a ausência de CAP, com exceção daqueles indivíduos com obesidade grau II, que, em grande parte, apresentaram CAP moderada. Assim, esses fatos podem sugerir que a presença de CAP ou TCAP podem ser contribuintes com o excesso de peso. Vale lembrar que na presente pesquisa as diferenças de médias de IMC conforme a presença ou ausência de CAP foi significativa.

Acerca da relação entre CAP e obesidade, em uma pesquisa que objetivou avaliar a qualidade de vida, imagem e satisfação corporal de pacientes obesos e sua associação com o Transtorno de Compulsão Alimentar Periódica, foi percebido que a presença do transtorno está associada com obesidade de maior gravidade e maiores variações de peso. Além disso, os obesos compulsivos apresentavam um hábito alimentar característico e específico para essa classe de pacientes ${ }^{(23)}$.

Alguns autores colocam que o TCAP pode estar associado com restrições dietéticas oriundas de preocupações que podem se desenrolar em futuros episódios compulsivos. $\mathrm{Na}$ atual pesquisa, indivíduos diagnosticados com CAP moderada e grave predominantemente consumiam mais de $2.000 \mathrm{Kcal} / \mathrm{dia}(70 \%$ e $66,67 \%$, respectivamente) e apresentaram médias de consumo energético maiores que pessoas sem CAP. Tais resultados assemelham-se ao encontrado em trabalho realizado com 110 pacientes com obesidade mórbida, de ambos os sexos, com idade entre 18 e 59 anos $^{(24)}$. Nesse estudo, o autor percebeu que todos os participantes da pesquisa seguiam uma dieta com mais de $1.500 \mathrm{Kcal} / \mathrm{dia}$, sugerindo então que a compulsão alimentar não está associada com restrições alimentares, e sim com um maior consumo dietético, podendo ser ocasionado pelos frequentes episódios compulsivos.

De acordo com os valores de referência propostos nas Dietary Referencie Intake (DRI) ${ }^{(25)}$, o consumo de carboidrato ideal deve estar entre $50 \%$ e $60 \%$ do Valor Calórico Total (VCT), o qual se encontra adequado para maior a parte dos entrevistados, independentemente destes apresentarem ou não a CAP. Entretanto, o consumo de fibras, cujo valor recomendado é de $20 \mathrm{~g}$ a $30 \mathrm{~g} / \mathrm{dia}$, mostrouse predominantemente inferior a $20 \mathrm{~g}$ por dia, também independentemente do diagnóstico de CAP, mas menor em portadores de CAP. Essa homogeneidade no perfil de consumo pode sugerir que isto pode caracterizar o padrão alimentar para indivíduos com excesso de peso, conforme foi detectado em estudo prévio ${ }^{(26)}$, com o objetivo de determinar a relação entre estado nutricional e a ingestão dietética em pacientes atendidos em uma clínica em Ipatinga-MG. Esta mostrou que o consumo médio de fibras de adultos com faixa etária entre 19 e 59 anos é de $18,25 \mathrm{~g}$ para indivíduos sobrepesados e 14,38g para os obesos, sugerindo que esse fato deve decorrer de uma alimentação a base de cereais refinados e pobre em frutas, verduras e cereais integrais.

Umapesquisarealizadacom 158 adultos costarriquenhos com faixa etária entre 20 e 64 anos e IMC maior que $25 \mathrm{Kg} /$ $\mathrm{m}^{2(27)}$ identificou consumo alimentar inadequado entre os entrevistados e que durante os episódios de compulsão, tal consumo era rico em gordura e carboidratos simples, o qual explica a falta de fibras na alimentação dos pacientes. Já em outro estudo com 50 pacientes submetidos à cirurgia bariátrica, com o objetivo de analisar os indícios de compulsão em seus entrevistados ${ }^{(28)}$, mostrou que os obesos compulsivos apresentavam um consumo maior de alimentos ricos em açúcar e gorduras quando comparados àqueles indivíduos sem compulsão.

Quanto ao consumo de lipídeos, recomenda-se uma ingestão de 10 a $35 \%$ do $\mathrm{VCT}^{(25)}$. Indivíduos com CAP moderada e grave apresentaram o consumo médio dentro do preconizado, porém, as médias, cujas diferenças não foram estatisticamente significativas, foram um pouco mais elevadas quando comparados com o da população total estudada, o que pode decorrer da maior ingestão calórica dos participantes com este diagnóstico e pode sugerir a 
existência de uma relação causa-efeito entre a compulsão e uma alimentação nutricionalmente inadequada.

Em um trabalho realizado com pacientes atendidos no Laboratório de Avaliação Nutricional (LAN) da Universidade Paulista, Campus Araçatuba-SP, de 20 a 65 anos de idade ${ }^{(29)}$, foi possível perceber que o consumo de carboidrato assemelha-se com o percebido no trabalho atual, uma vez que encontra-se dentro dos níveis preconizados. Por outro lado, indivíduos com CAP moderada e grave demostraram consumir uma quantidade menor de lipídios em gramas, quando relacionados com aqueles sem CAP. O contrário sendo visto quando relacionado a esse lipídio com o total de calorias consumidas, embora não confirmado aqui estatisticamente. A ausência de significância pode resultar do número bem menor de indivíduos diagnosticados com CAP, comparado aos sem CAP. Entretanto, considerando o perfil encontrado de consumo de lipídios, em gramas, em detrimento da análise estatística, o menor consumo de lipídio entre aqueles com CAP pode ter decorrido da omissão do consumo real pelos indivíduos diagnosticados com CAP, podendo assim ter subestimado o real consumo.

Portanto, percebe-se que aqueles pacientes com graus de obesidade mais elevados apresentaram uma maior prevalência de CAP moderada e grave. Nota-se ainda que esses mesmos indivíduos possuam um consumo alimentar acima da média da maioria das pessoas, o que pode ser exemplificado pelo consumo calórico mais elevado, bem como uma dieta desequilibrada em se tratando dos macronutrientes, uma vez que apresentaram um maior consumo de lipídeos quando comparados aos entrevistados com níveis de IMC mais baixos.

Algumas limitações foram percebidas ao longo da pesquisa, dentre as quais a resistência da população alvo em participar, uma vez que era necessário longo tempo para que a pesquisa pudesse ser concluída. Percebeu-se também que a maioria das pessoas não se sentia à vontade com o assunto, o que levava muitas vezes à omissão das respostas. Além disso, ainda existem poucos estudos disponíveis sobre CAP, mesmo fora do Brasil.

Como sugestão para estudos futuros, pode-se citar a expansão da amostra, como forma de possibilitar análises mais confiáveis e a investigação da associação entre a presença de compulsão e a submissão ou ausência de submissão, em momentos anteriores, a métodos para indução da perda de peso, averiguando se esse poderia ser um motivo para que o indivíduo viesse a desenvolver a compulsão.

Não desprezando os $23 \%$ da amostra diagnosticada com CAP, para tratar o comportamento compulsivo pode ser necessário acompanhamento multiprofissional, por exemplo, psicoterápico, nutricional e por profissional de educação física. Sabendo que a obesidade é uma condição física multifatorial, o tratamento da compulsão alimentar certamente trará benefícios para o controle da doença, uma vez que o consumo exagerado de calorias é umas das principais causas para a obesidade.

\section{CONCLUSÃO}

Um terço dos participantes apresentou algum grau de compulsão alimentar relacionado com alteração de estado nutricional, destacando que os participantes com compulsão alimentar periódica moderada e grave apresentaram um padrão alimentar com mais calorias e menos fibras.

\section{REFERÊNCIAS}

1. Cortez CM, Araújo EA, Ribeiro MV. Transtorno de compulsão alimentar periódico e obesidade. ACM Arq Catarin Med.2011;40(1):96-104.

2. Venzon $\mathrm{CN}$, Alchieri JC. Indicadores de compulsão alimentar periódica em pós-operatório de cirurgia bariátrica. Psico (Porto Alegre). 2014;45(2):239-49.

3. Calegari K. Associação entre compulsão alimentar e sedentarismo: fatores que levam a obesidade. RBONE Rev Bras Obes. 2012;6(35):242-53.

4. Teixeira PC, Costa RF, Matsudo SMM, Cordás TA. A prática de exercícios físicos em pacientes com transtornos alimentares. Rev Psiq Clín. 2009;36(4): $145-52$.

5. Nunes RM. Transtorno da compulsão alimentar periódica (TCAP) e a abordagem da Terapia Cognitiva Comportamental (TCC). Rev Psiquiatr. 2012;25(3):166-70.

6. Melo BJ, Neves SMM. Modelo cognitivocomportamental para tratamento de crianças obesas com compulsão alimentar periódica. Fragm Cult. 2014;24:73-83.

7. Pivetta LA, Silva RMVG. Compulsão alimentar e fatores associados em adolescentes de Cuiabá, Mato Grosso, Brasil. Cad Saúde Pública. 2010;26(2):337-46.

8. Passos TCBM, Yazigi L, Claudino AM. Aspectos ideativos no transtorno da compulsão alimentar periódica: estudo com o Rorschach. Psico USF. 2008;13(1):69-74.

9. Chaves L, Navarro AC. Compulsão alimentar, obesidade e emagrecimento. RBONE Rev Bras Obes. 2011;5(27):110-20.

10. Ministério da Saúde (BR), Secretaria de Atenção à Saúde. Orientação Para Coleta e Análise de Dados 
Antropométricos em Serviço de Saúde. Brasília: Ministério da Saúde; 2011.

11. Triola MF. Introdução a estatística. $7^{\mathrm{a}}$ ed. Rio de Janeiro: LTC; 1999.

12. Freitas S, Lopes CS, Coutinho W, Appolinário JC. Tradução e adaptação para o português da Escala de Compulsão Alimentar Periódica. 2001. Rev Bras Psiquiatr. 2001;23(4):215-20.

13. Fisberg RM, Slater B, Marchioni DML, Martini LA. Inquéritos alimentares: métodos e base científica. São Paulo: Manole; 2005.

14. Alvarez BR, Pavan AL. Alturas e comprimentos. In: Petroski EL, organizador. Antropometria: técnicas e padronizações. Porto Alegre: Pallotti, 1999. p. 29-51.

15. World Health Organization - WHO. Physical status: the use and interpretation of anthropometry. Report of a WHO Expert Committee. Geneva: WHO; 1995. (Technical Report Series, 854).

16. Pinheiro ABV, Lacerda EMA, Benzecry EH, Gomes MCS, Costa VM. Tabela para avaliação do consumo alimentar em medidas caseiras. $5^{\text {a }}$. ed. São Paulo: Atheneu; 2009.

17. Núcleo de Estudos de Composição de Alimentos (BR), Universidade de Campinas. Tabela brasileira de composição de alimentos. 4. ed. Campinas: NEPAUNICAMP; 2011.

18. Castro MLD. Associação do polimorfismo rs9939609 do FTO ao transtorno de compulsão alimentar periódica (TCAP) em pacientes obesos mórbidos [dissertação]. Porto Alegre: Universidade Federal do Rio Grande do Sul-Faculdade de Medicina; 2013

19. Mascarenhas MTL, Almeida MMG, Araújo TM, Prisco APK. Transtornos alimentares na população de 20 a 59 anos de Feira de Santana (BA), 2007. Cad Saúde Colet (Rio J). 2011;19(2):179-86.

20. Prisco APK, Araújo TM, Almeida MMG, Santos KOB. Prevalência de transtornos alimentares em trabalhadores urbanos de município do Nordeste do Brasil. Ciênc Saúde Coletiva. 2013;18(4):1109-18.

21. Palavras MA, Kaio GH, Mari JJ, Claudino AM. Uma revisão dos estudos latino-americanos sobre o transtorno da compulsão alimentar periódica. Rev Bras Psiquiatr. 2011;33(Supl 1):581-96.

22. Tramontt CR, Schneider CD, Stenzel LM. Compulsão alimentar e bulimia nervosa em praticantes de exercício físico. Rev Bras Med Esporte. 2014;20(5):383-7.
23. Melo MMO. Compulsão alimentar, imagem corporal e qualidade de vida em crianças e adolescentes obesos [dissertação]. Belo Horizonte: Faculdade de Medicina da Universidade Federal de Minas Gerais; 2011.

24. Abilés V, Ruiz SR, Abilés J, Obispo A, Gandara N, Luna V, et al. Effectiveness of cognitive-behavioral therapy in morbidity obese candidates for bariatric surgery with and without binge eating disorder. Nutr Hosp. 2013;28(5):1523-9.

25. Institute Medicine(US).PanelOnMicronutrients dietary Reference Intakes for Energy, Carbohydrate, Fiber, Fat, Fatty Acids, Cholesterol, Protein and Amino Acids. Panel on Macronutrients Panel on the Definition of Dietary Fiber, Subcommittee on Upper Reference Levels of Nutrients, Subcommittee on Interpretation and Uses of Dietary Reference Intakes, and the Standing Committee on the Scientific Evaluation of Dietary Reference Intakes, Food and Nutrition Board. Washington: National Academies Press; 2005.

26 .Pereira LSS, Pinto RCP, Azevedo V, Muzi, VR, Quintão D. Relação entre perfil antropométrico e a ingestão dietética em pacientes atendidos na clínica escola da Faculdade Pitágoras, Campus IpatingaMG. RBONE Revista Brasileira de Obesidade, Nutrição e Emagrecimento. 2012;6(31):4-12.

27. Coronado ALC, Brenes ALR. prevalencia de manifestaciones del trastorno por atracónen adultos con sobrepeso y obesidad, Costa Rica. Rev Costarric Salud Pública. 2013;22(1):20-6.

28. Machado CE, Zilberstein B, Cecconello I, Monteiro M. Compulsão alimentar antes e após a cirurgia bariátrica. ABCD Arq Bras Cir Dig. 2008;21(4): 185-91.

29. Mosca LN, Costa LRLG, Ramos CFC, Asano LMT, Ferreira AD. Compulsão alimentar periódica de pacientes em tratamento para redução de peso. J Health Sci Inst. 2010;28(1):59-63.

\section{Endereço para correspondência:}

Bruna Yhang da Costa Silva

Instituto Federal de Educação, Ciência e Tecnologia do

Ceará - IFCE

Rua Estevão Remígio de Freitas, 1145

Bairro: Centro

CEP: 62930-000 - Limoeiro do Norte - CE - Brasil

E-mail: brunayhang@gmail.com 